\title{
Font size modulates saccade-target selection in Chinese reading
}

\author{
Hua Shu • Wei Zhou • Ming Yan • Reinhold Kliegl
}

Published online: 6 November 2010

(C) Psychonomic Society, Inc. 2010

\begin{abstract}
In alphabetic writing systems, saccade amplitude (a close correlate of reading speed) is independent of font size, presumably because an increase in the angular size of letters is compensated for by a decrease of visual acuity with eccentricity. We propose that this invariance may (also) be due to the presence of spaces between words, guiding the eyes across a large range of font sizes. Here, we test whether saccade amplitude is also invariant against manipulations of font size during reading Chinese, a character-based writing system without spaces as explicit word boundaries for saccade-target selection. In contrast to word-spaced alphabetic writing systems, saccade amplitude decreased significantly with increased font size, leading to an increase in the number of fixations at the beginning of words and in the number of refixations. These results are consistent with a model which assumes that word beginning (rather than word center) is the default saccade target if the length of the parafoveal word is not available.
\end{abstract}

Keywords Eye movement · Saccade $\cdot$ Chinese $\cdot$ Font size

H. Shu $\cdot$ W. Zhou

State Key Laboratory of Cognitive Neuroscience and Learning, Beijing Normal University,

Beijing, People's Republic of China

M. Yan $(\square)$

Department of Psychology, Peking University,

Yiheyuan Road 5th,

100871 Beijing, People's Republic of China

e-mail: mingyan@pku.edu.cn

M. Yan · R. Kliegl

Department of Psychology, University of Potsdam,

Potsdam, Germany

\section{Introduction}

One intriguing perceptual phenomenon related to reading is that text-based or relative saccade amplitude measured in number of letters that a saccade covers apparently does not depend on font size - at least within the usual range. Saccade amplitude regulates the number of fixations and, therefore, is a close correlate of reading speed. Arguably, the reason for this invariance is that the increase in visibility associated with large font size is (almost perfectly) traded off against a decrease of visual acuity associated with the font size-related shift of letters and words towards more eccentric positions relative to the fixation position (O'Regan, 1990). Consequently, number of letters (rather than angular size) is used as the metric of saccade amplitudes in reading research. One limitation associated with these results, however, is that the invariance of relative saccade amplitude for different font sizes has been demonstrated only for spaced alphabetic scripts. Spaces, however, provide a very powerful, low-spatial frequency demarcation of parafoveal word boundaries for the usual range of font sizes. With precise word boundaries, saccades can be programmed for the word centers, allowing readers to maintain their normal reading style under a variety of font sizes. Conversely, if precise word boundaries are not available, like in writing systems without spaces between words, the change in saccade target selection may reveal the effects of font size.

In this article, we ask whether font size modulates the selection of saccade targets in reading Simplified Chinese. ${ }^{1}$

\footnotetext{
${ }^{1}$ There are two sets of modern Chinese writing systems: the Simplified and the Traditional Chinese. The former one is mainly used in Mainland China where there was a reduction in the visual complexity of many characters in the $1950 \mathrm{~s}$, and the latter is mainly used in Taiwan and Hong Kong.
} 
The expectation is that font-size invariance may not hold for this writing system due to the lack of spaces between words and the effects of this difference on the selection of saccade targets (Yan, Kliegl, Richter, Nuthmann, \& Shu, 2010). Yan et al. (2010) argued that, depending on failure or success with segmentation of parafoveal word boundaries, readers of Chinese dynamically select either the beginning or the center of words as saccade targets. This proposal was supported empirically by a strong tendency to fixate at the word center in single-fixation cases and at the word beginning in multiple-fixation cases. Moreover, the preferred viewing location (PVL; Rayner, 1979) of the first fixation in multiple-fixation cases was at the word beginning, similar to what had been reported for reading of unspaced English text (Rayner, Fischer, \& Pollatsek, 1998) and Japanese text written without word boundaries (Kajii, Nazir, \& Osaka, 2001; Sainio, Hyönä, Bingushi, \& Bertram, 2007). In summary, if the end of the next word is not known, the beginning of the next word may be the most informative region and therefore be selected as the primary saccade target.

In contrast, research on eye-movement control in reading of spaced alphabetic scripts strongly suggests that saccades are directed at the word center with the peak of a Gaussian distribution of landing positions slightly left of word center (Rayner, 1979). Saccades do not land precisely at their intended target location due to systematic range error associated with the saccade amplitude and with noise in the perceptual or oculomotor system (McConkie, Kerr, Reddix, \& Zola, 1988). Recently, Engbert and Krügel (2010) showed that the saccadic range error associated with variation of within-word fixation positions can be derived as the result of saccade programming according to a Bayesian model that optimally reconciles visual and oculomotor processing constraints. In summary, when reading spaced alphabetic script, word centers are generally taken to serve as the basic unit for saccade targeting during reading (McConkie et al., 1988; O'Regan \& Levy-Schoen, 1987; Reichle, Rayner, \& Pollatsek, 1999; but see also Yang \& McConkie, 2004).

How does saccade amplitude depend on the angular size of letters? Several studies on reading of spaced alphabetic scripts demonstrated constant relative saccade amplitude for different viewing distances. Morrison and Rayner (1981) found relative saccade amplitude did not differ at viewing distances of 36,53 and $71 \mathrm{~cm}$, which corresponded to angular sizes of $0.69^{\circ}, 0.47^{\circ}$ and $0.35^{\circ}$ per letter respectively. O'Regan (1983) reported that the relative saccade amplitude was not affected by six different viewing distances from 25 to $75 \mathrm{~cm}$. Bullimore and Bailey (1995) also reported little change in the number of letters read per forward saccade for normal readers. Recently, Miellet, O'Donnell, and Sereno (2009) compared the difference in absolute saccade amplitude (i.e., defined in number of pixels) and relative saccade amplitude measurements between normal reading and gaze-contingent parafoveal magnification (PM) conditions, in which the size of parafoveal letters were enlarged as a function of their eccentricity from current fixation. They found that the absolute saccade amplitude for PM was significantly longer than that for normal texts, but the relative saccade amplitude was similar between these two conditions, replicating results from earlier studies. The only exception to the general pattern was that saccades were longer for a magnification factor of 7.5 compared to one of 3 in a study by Mohammed and Dickinson (2000) in which participants were wearing magnifiers during reading.

Obviously, word-based targeting depends on the availability of information about the beginning and the end of the target word. The spaces between words in alphabetic scripts provide exactly this information. Removing space information not only reduces reading speed significantly (Epelboim, Booth, Ashkenazy, Taleghani, \& Steinman, 1997; Epelboim, Booth, \& Steinman, 1994; Rayner et al., 1998; Rayner \& Pollatsek, 1996), but more importantly, the PVL curve decreases sharply and linearly from the beginning to the end of the word, because it presumably impedes the identification of the beginning and end of a given word which is necessary to delineate the saccade target (Rayner et al., 1998; Rayner \& Pollatsek, 1996).

Here, we propose that the strong signal associated with word boundary (i.e., visually low spatial frequency information of the space between words) may actually contribute to the independence of font size and relative saccade amplitude as far as saccade target selection in reading of alphabetic scripts is concerned (O'Regan, 1979, 1980; Rayner, 1979). Therefore, we manipulated the font size of Chinese sentences to check whether the invariance of relative saccade amplitude also holds for a writing system that does not use spaces to separate words.

The Chinese script uses characters with square-shaped forms of different levels of visual complexity as the basic writing units; they occupy the same amount of horizontal extent irrespective of their visual complexity. One of the most apparent characteristics of Chinese script is the lack of spaces between words or other explicit information indicating word boundaries in orthography. For example, Hsu and Huang (2000a, 2000b) observed benefits of reading speed by insertion of inter-word spaces for difficult and ambiguous sentences. Bai, Yan, Liversedge, Zang, and Rayner (2008) slowed reading by inserting spaces that generated nonwords. These studies demonstrated the relevance of word units in the script and suggest that parafoveal word-boundary information is used for target selection. 
The present study is a follow-up and an extension of Yan et al. (2010) to test the effects of visual acuity limits on eyemovement control of saccade target selection in unspaced Chinese reading by using sentences presented with different font sizes. Given that Chinese is a language written without explicit word boundaries and that, therefore, readers are assumed to segment words in the parafovea, we expect that visual acuity plays a more important role here than in alphabetic languages: When sentences are presented with larger fonts, the visual acuity drop might cause a lack of information about the endings of the words to be fixated next. This situation, in turn, is likely to impede successful parafoveal word segmentation. As a consequence of such segmentation failures or delays, a large percentage of firstof-multiple fixations is expected to occur at the word beginning.

\section{Method}

Subjects

Forty-eight undergraduate or graduate students from Beijing Normal University with normal or corrected to normal vision (above 0.1 in $\mathrm{E}$ test for visual acuity), who were native speakers of Chinese, participated in the eye-tracking experiment. The sessions lasted $30-40 \mathrm{~min}$. Subjects received $10 \mathrm{CNY}$ for their participation.

\section{Material}

Subjects read 120 sentences from the Beijing Sentence Corpus (Yan et al., 2010). The sentences were selected from a local newspaper, and some of them were slightly edited to avoid possible syntactic, semantic or word-boundary ambiguities. By manipulating the font sizes while keeping the viewing distance constant among conditions, four angular size conditions $\left(0.4^{\circ}, 0.7^{\circ}, 1.4^{\circ}\right.$, and $2.1^{\circ}$ per Chinese character, corresponding to $12,20,40$, and 60 pix font, respectively) were adopted in the present study.

Sentences used in this experiment were 15-19 characters in length $(M=17.9, \mathrm{SD}=1.2)$, corresponding to $7-12$ words $(M=9.7, \mathrm{SD}=1.0)$. These sentences comprise 1,158 tokens of 713 words (types); 6.7, 85.7, 5.6, and $2.0 \%$ of the words (types) are 1-4 characters long, respectively. This word-length distribution is representative for Chinese sentences (Yu, Zhang, Jing, Peng, Zhang, \& Simon, 1985). The number of strokes per word, which is a rough index of its visual complexity, varies form 2 to 42 ( $M=$ $15.5, \mathrm{SD}=5.5$ ); the percentages of number of strokes in the range of $1-10,11-20$, and above 20 are 17,67 , and $16 \%$, respectively. Word frequencies were taken from the Modern Chinese Word Frequency Dictionary (Beijing Language
Institute Publisher, 1986) based on 1.2 million words. Occurrences of words varied from 1 to $65,671(M=577$, $\mathrm{SD}=2,945)$ per 1.2 million. The percentages of words with frequencies in the range of less than 10, 10-100, 100 1,000 , and above 1,000 per million words are $15,37,39$, and $9 \%$, respectively.

\section{Apparatus}

Eye movements were calibrated and recorded binocularly with an EyeLink II system $(500 \mathrm{~Hz})$. Sentences occupied only one line on the screen and were presented one at a time at the $1 / 3$ vertical position from the top of the screen of a 21-inch $($ c. $53.3 \mathrm{~cm})$ Dell P1130 CRT Monitor $(1,280 \times$ 1,024 resolution; frame rate $100 \mathrm{~Hz}$ ) controlled by a $\mathrm{P} 4$ computer running at $2.8 \mathrm{GHz}$ under a Windows XP environment. Subjects were seated $50 \mathrm{~cm}$ in front of the monitor with the head positioned on a chin rest.

\section{Procedure}

Subjects were calibrated with a standard nine-point grid for both eyes. After validation of calibration accuracy, a fixation point appeared on the left side of the monitor. If the eye tracker identified a fixation on the fixation spot, the fixation point disappeared and a sentence was presented such that the center of the first character in the sentence appeared at the fixation point position.

Subjects were instructed to read the sentences for comprehension, then fixate a dot in the lower right corner of the monitor, and finally press a button on a joystick to signal the completion of a trial. The sentence was replaced by an easy yes-no question pertaining to the current sentence on 28 trials (23\%), which the participant answered with two different joystick buttons. These questions served primarily to ensure reading for comprehension. Participants correctly answered $93 \%$ of all questions ( $\mathrm{SD}=6 \%$ ); font size had no significant effect on accuracy $(F(3,141)=1.65$, $p>.1)$. Subsequent to a response, fixation of a fixation point initiated presentation of the next sentence or a drift correction. The experimenter carried out an extra calibration if the tracker did not detect both eyes within a predefined window of $1.5^{\circ} \times 1.5^{\circ}$ around the initial fixation point. The four font-size conditions (30 sentences in each) were blocked and counterbalanced over subjects and sentences.

\section{Data analysis}

Analyses were based on software originally developed for the analysis of the Potsdam Sentence Corpus reading data (e.g., Kliegl, Grabner, Rolfs, \& Engbert, 2004) and further updated for the analysis of Beijing Sentence Corpus reading 
data (Kliegl et al., 2009; Yan et al., 2010). Fixations were determined with an algorithm for binocular saccade detection introduced by Engbert and Kliegl (2003). In a slight modification, we fixed the threshold for saccade detection at a fixed value for each subject based on the mean threshold computed across the four experimental conditions.

Sentences containing a blink or loss of measurement were deleted (i.e., 4\%). Analyses were based on fixations measured in the right eye. Fixations were horizontally allocated to zones representing $50 \%$ of a character. For example, a Chinese two-character word comprises 4 zones, numbered from 1 to 4 sequentially. First- and singlefixation durations as well as gaze durations with firstfixation durations shorter than $60 \mathrm{~ms}$ or longer than $600 \mathrm{~ms}$ were excluded, retaining $99 \%$ of all fixations. First-fixation duration is the duration of the initial fixation on a word irrespective of number of fixations on the word; singlefixation duration is the duration of fixation on a word that is fixated only once; gaze duration is the sum of all first-pass fixations on a word before making a saccade to another word.

\section{Results}

\section{Global analyses}

In this section, we present the mean and standard deviation of reading speed (in number of characters per minute), absolute saccade amplitude (in pixels), relative saccade amplitude (in characters) and fixation duration (in milliseconds) in Table 1a, as well as percentage of different saccade types in Table $2 \mathrm{a}$. Inferential statistics are based on planned comparisons (contrasts) between two neighboring font size conditions; they are reported in Tables $1 \mathrm{~b}$ and $2 \mathrm{~b}$. Estimates are from a linear mixed model (LMM) with crossed random effects for subjects and items using the lmer function of the lme 4 package (Bates, Maechler, \& Dai, 2008) in the $\mathrm{R}$ environment for statistical computing and graphics (R Core Development Team, 2008). Estimates larger than $2 \mathrm{SE}$ (i.e., $t>2$ ) are interpreted as significant. Repeated measure analysis of variance yielded the same pattern of significance.

Reading speed The reading speed differed significantly between the three neighboring contrasts (Table 1b), with a maximum speed when sentences were presented with an angular size of $0.7^{\circ}$ per character. Reading speed demonstrated an inverted- $U$ curve as a function of angular size, as also reported in $\mathrm{Xu}$ and Jordan (2009).

Forward saccade amplitude The forward saccade amplitude is reported both in absolute and relative measures. Significant differences were found between neighboring conditions with absolute saccade amplitude increasing with font size (Table 1b), replicating results from alphabetic reading studies (e.g., Morrison \& Rayner, 1981). However, relative saccade amplitude significantly decreased with increasing font size (Fig. 1 and Table 1b). We take this result as evidence for a variation of saccade types and saccade targeting as a function of font size.

Fixation duration The mean fixation duration also decreased with increased font size (Table 1b), similar to relative saccade amplitude. The decrease of fixation duration with increased character angular size replicates results from alphabetic reading by Morrison and Rayner (1981) and Bullimore and Bailey (1995).
Table $1(A)$ means (and standard deviations) for reading speed (in number of characters per minute), absolute saccade amplitude (in pixels) and text-based relative saccade amplitude (in number of characters) and fixation duration (in milliseconds); $(B)$ contrast estimates and their associated $t$ values

\begin{tabular}{|c|c|c|c|c|c|c|c|c|c|}
\hline & & \multicolumn{2}{|c|}{ Reading speed } & \multicolumn{2}{|c|}{ Abs. saccade amplitude } & \multicolumn{2}{|c|}{ Rel. saccade amplitude } & \multicolumn{2}{|c|}{ Fixation duration } \\
\hline \multicolumn{10}{|l|}{ (A) } \\
\hline \multirow[t]{4}{*}{ Angular Size } & $0.4^{\circ}$ & \multicolumn{2}{|c|}{$443(164)$} & \multicolumn{2}{|c|}{$30(8)$} & \multicolumn{2}{|c|}{$2.5(0.7)$} & \multicolumn{2}{|c|}{$296(47)$} \\
\hline & $0.7^{\circ}$ & \multicolumn{2}{|c|}{$478(217)$} & \multicolumn{2}{|c|}{$43(10)$} & \multicolumn{2}{|c|}{$2.2(0.5)$} & \multicolumn{2}{|c|}{$256(41)$} \\
\hline & $1.4^{\circ}$ & \multicolumn{2}{|c|}{445 (124) } & \multicolumn{2}{|c|}{$78(16)$} & \multicolumn{2}{|c|}{$2.0(0.4)$} & \multicolumn{2}{|c|}{$229(33)$} \\
\hline & $2.1^{\circ}$ & \multicolumn{2}{|c|}{$414(98)$} & \multicolumn{2}{|c|}{$114(23)$} & \multicolumn{2}{|c|}{$1.9(0.4)$} & \multicolumn{2}{|c|}{$217(32)$} \\
\hline \multicolumn{10}{|l|}{ (B) } \\
\hline & & Est. & $t$ & Est. & $t$ & Est. & $t$ & Est. & $t$ \\
\hline \multirow[t]{3}{*}{ Contrast } & $0.4-0.7^{\circ}$ & 32.9 & 8.0 & 12.9 & 27.1 & -.31 & -16.5 & -38.9 & -26.2 \\
\hline & $0.7-1.4^{\circ}$ & -31.3 & -7.6 & 33.8 & 74.2 & -.19 & -10.4 & -28.3 & -19.7 \\
\hline & $1.4-2.1^{\circ}$ & -32.6 & -7.8 & 34.6 & 78.8 & -.05 & -2.9 & -11.5 & -8.2 \\
\hline
\end{tabular}

Means and standard deviations are computed across subjects' means 
Table $2(A)$ The percentage of one-word forward, skipping, intraword forward, intraword backward and regressive saccades; $(B)$ contrast estimates and their associated $t$ values

Saccade type

One-word forward $\quad$ Skipping $\quad$ Intraword forward $\quad$ Intraword backward $\quad$ Regressive

(A)

\begin{tabular}{|c|c|c|c|c|c|c|c|c|c|c|c|}
\hline \multirow[t]{4}{*}{ Angular Size } & $0.4^{\circ}$ & \multicolumn{2}{|l|}{$46(10)$} & \multicolumn{2}{|c|}{$29(15)$} & \multicolumn{2}{|l|}{$14(6)$} & \multicolumn{2}{|l|}{$4.0(2)$} & \multicolumn{2}{|l|}{$6.7(4)$} \\
\hline & $0.7^{\circ}$ & \multicolumn{2}{|l|}{$51(9)$} & \multicolumn{2}{|l|}{$26(14)$} & \multicolumn{2}{|l|}{$14(6)$} & \multicolumn{2}{|l|}{$3.9(2)$} & \multicolumn{2}{|l|}{$5.2(4)$} \\
\hline & $1.4^{\circ}$ & \multicolumn{2}{|l|}{$54(7)$} & \multicolumn{2}{|c|}{$20(11)$} & \multicolumn{2}{|l|}{$19(7)$} & \multicolumn{2}{|l|}{$3.3(2)$} & \multicolumn{2}{|l|}{$3.4(3)$} \\
\hline & $2.1^{\circ}$ & \multicolumn{2}{|l|}{$52(7)$} & \multicolumn{2}{|l|}{$19(10)$} & \multicolumn{2}{|l|}{$21(7)$} & \multicolumn{2}{|l|}{$2.7(2)$} & \multicolumn{2}{|l|}{$5.2(3)$} \\
\hline & & Est. & $t$ & Est. & $t$ & Est. & $t$ & Est. & $t$ & Est. & $t$ \\
\hline \multirow[t]{3}{*}{ Contrast } & $0.4-0.7^{\circ}$ & .053 & 7.7 & -.037 & -6.7 & -.001 & -0.2 & -.002 & -0.8 & -.014 & -5.1 \\
\hline & $0.7-1.4^{\circ}$ & .029 & 4.3 & -.062 & -11.3 & .054 & 14.1 & -.004 & -1.7 & -.018 & -6.5 \\
\hline & $1.4-2.1^{\circ}$ & -.023 & -3.3 & -.010 & -1.8 & .022 & 5.8 & -.005 & -2.3 & .016 & 5.8 \\
\hline
\end{tabular}

Means and standard deviations are computed across participants' means

Mean proportions for different saccade types

The significant difference in relative saccade amplitude reported above implied a variation of percentages of different types of saccades as a function of font size. Table 2 presents the percentage of one-word forward, skipping, intraword forward, intraword backward and regressive saccades. The percentage of one-word forward saccades peaked in the $1.4^{\circ}$ condition; the percentage of regressions mirrored this pattern in the reverse. A similar compensation was observed for the percentage of intraword forward and backward saccades, but the overall percentage of refixations (i.e., sum of intraword forward and backward saccades) increased with font size from $0.7^{\circ}$ to $2.1^{\circ}$ conditions $(b=.050, \mathrm{SE}=.004, t=11.5$ and $b=.017$, $\mathrm{SE}=.004, t=3.9$, for $0.7-1.4^{\circ}$ and $1.4-2.1^{\circ}$ comparisons, respectively). Furthermore, the change associated with

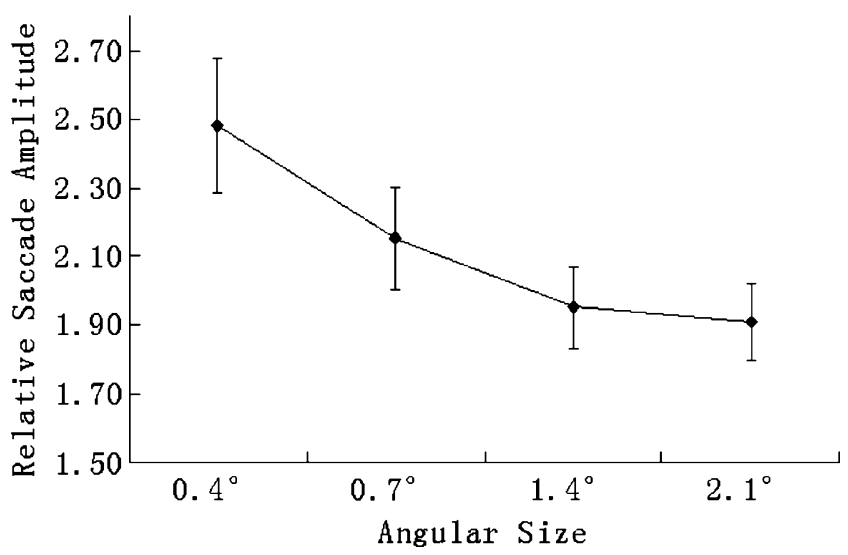

Fig. 1 Relative saccade amplitude with $95 \%$ confidence intervals as a function of angular size. Means and confidence intervals are computed across subjects' means forward refixations was more pronounced than that with backward refixations. Note that such an asymmetry is difficult to explain with reference to mere random saccadic errors or mislocated fixations. Finally, the percentage of skipping decreased with font size.

\section{Landing position analyses}

Only data of two-character words were included in the following analyses due to the high percentage of words of this length in the experimental material and in Chinese texts in general (Yu et al., 1985). First fixation landing positions shifted towards word beginning for the larger font size conditions as compared to the smaller font size conditions $(b=.002, \mathrm{SE}=.010, t=0.2 ; b=-.052, \mathrm{SE}=.010$, $t=-5.2 ; b=.002, \mathrm{SE}=.010, t=0.3$, for $0.4-0.7^{\circ}, 0.7-1.4^{\circ}$ and $1.4-2.1^{\circ}$ comparisons, respectively). Figure 2 displays proportions of single fixations (top) and first-of-two fixations (bottom) for two-character words as a function of initial landing position (i.e., the preferred viewing location curve, PVL; Rayner, 1979).

Single fixation PVL For each font size the probability of fixations differed between character zones $[F(2,94)=4.3$, $p<.05 ; F(2,94)=12.5, p<.001 ; F(2,94)=23.9, p<$ $.001 ; F(2,94)=24.1, p<.001$; for $0.4-2.1^{\circ}$ conditions, respectively]. To test whether there is a significant peak in each curve (visible in Fig. 2a), we specified linear and quadratic trends across character zone, leaving out the final zone to avoid a singular design matrix. Curvature is established with a significant negative quadratic trend. This was the case for all font sizes: For $0.7^{\circ}, 1.4^{\circ}$ and $2.1^{\circ}$ conditions, both linear and quadratic trends were reliable [linear trends: $F(1,47)=4.8, p<.05 ; F(1,47)=31.3$, 

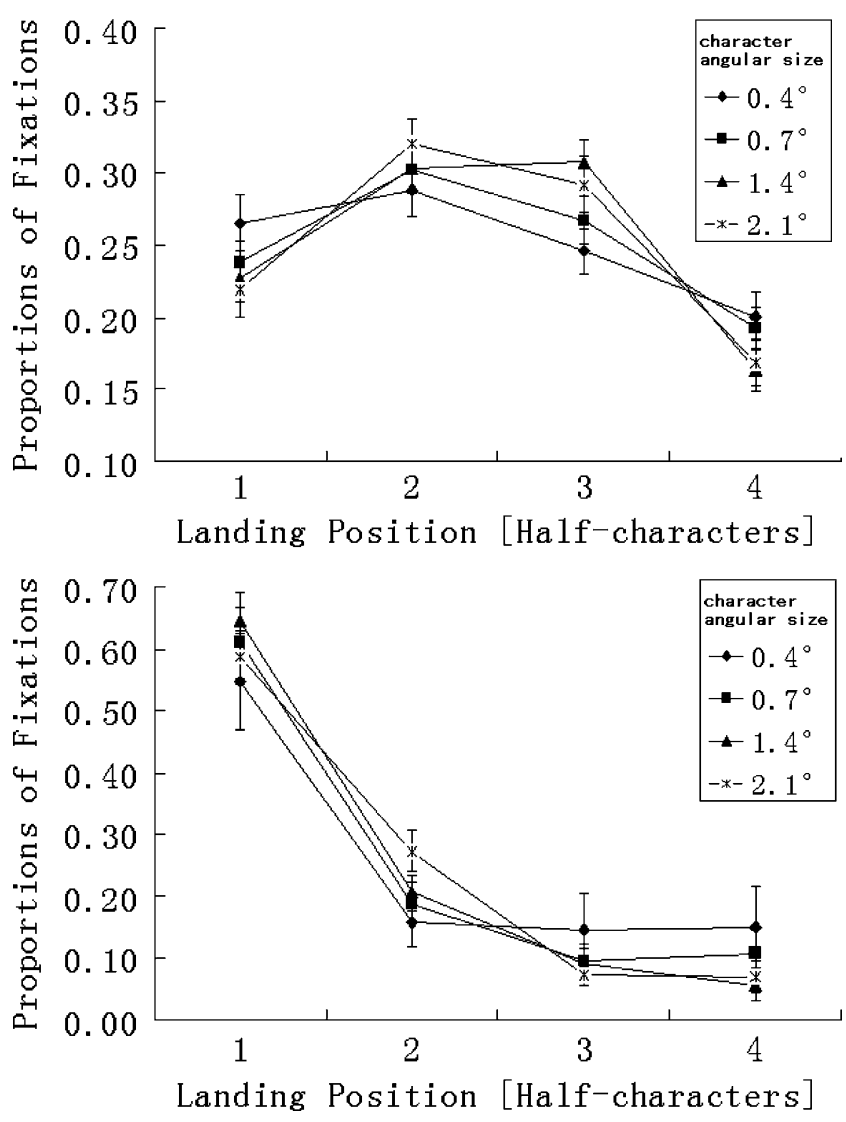

Fig. 2 Landing position distributions of single fixations (top) and first fixation in two-fixation cases (bottom) in 2-character words with $95 \%$ confidence intervals

$p<.001 ; F(1,47)=17.9, p<.001$; for $0.7-2.1^{\circ}$ conditions, respectively; quadratic trends: $F(1,47)=22.6, p<.001$; $F(1,47)=12.8, p<.001 ; F(1,47)=35.3, p<.001$; for $0.7-2.1^{\circ}$ conditions, respectively]. For the $0.4^{\circ}$ condition, only the quadratic trend was significant $[F(1,47)=7.7, p<$ $.01]$ but not the linear trend $(p>.1)$.

First-of-two fixation PVL For each condition, the probability of fixations differed among half-character zones $[F(2,94)=$ $43.7, p<.001 ; F(2,94)=125.2, p<.001 ; F(2,94)=200.7$, $p<.001 ; F(2,94)=180.1, p<.001 ;$ for $0.4-2.1^{\circ}$ conditions]. For these curves, we want to establish that the proportion of first fixation decreases from the beginning to the end, potentially reaching a plateau in the middle of the word (see Fig. 2b). The shape of these curves translates into significant negative linear trends and significant positive quadratic trends. Indeed, these predictions were met for all font sizes [linear trends: $F(1,47)=45.7, p<.001 ; F(1,47)=$ $172.8, p<.001 ; F(1,47)=280.8, \mathrm{p}<.001 ; F(1,47)=$ $422.8, p<.001$; for $0.4-2.1^{\circ}$ conditions, respectively; quadratic trends: $F(1,47)=38.0, p<.001 ; F(1,47)=$ $42.0, p<.001 ; F(1,47)=55.5, p<.001 ; F(1,47)=5.0, p<$ .05 ; for $0.4-2.1^{\circ}$ conditions, respectively]. The result replicates Yan et al. (2010).
Effects of visual complexity Visual complexity of Chinese characters is of primary importance for character recognition (e.g., Liu, Klein, Xue, Zhang, \& Yu, 2009). Given our interest in font size, we chose a counterbalanced design in which all sentences appeared in every condition when averaged across all subjects to avoid a confound of visual complexity and font size. We can still test the effect of visual complexity by including it as a covariate in (generalized) linear mixed models and test its interaction with contrasts between font sizes. Visual complexity had the expected main effects on first-fixation landing position and refixation probability. For visually complex words, the first-fixation landing position shifted to the left (LMM: $b=-.0022, \mathrm{SE}=.0009, t=-2.6)$ and refixations were more likely (GLMM: $b=.0105, \mathrm{SE}=.0044, z=2.4$, $p<.05)$. Importantly, none of the interactions between visual complexity and font size was significant (all $t$ values $<1$ and $p$ values $>.1$ ).

\section{Discussion}

For spaced alphabetic script, relative saccade amplitude does not depend on font size. The main explanation for this invariance has been that an increase in the angular size of letters is compensated by a decrease of visual acuity with eccentricity. We considered it plausible that this invariance may (also) be due to the spaces between words that deliver precise information about parafoveal word boundaries for the entire range of usual font sizes. This assumption can be tested with reading of an unspaced writing system like Simplified Chinese. As expected, font size did matter in this case. Thus, unlike in alphabetic reading, the relative saccade amplitude in Chinese reading is modulated by font size. We derived this prediction from a recent model about flexible saccade targeting (Yan et al., 2010). We will first discuss the implications of the present study for this model and then turn to their implications for other theoretical issues relating to eye-movement control in reading.

Visual acuity modulates saccade-target selection

While the effect of font size on reading speed has been widely discussed in the literature, little is known about how font size modulates eye-movement control in Chinese reading, which is a language without explicit word boundaries guiding the eyes through text. The present study demonstrated that the number of refixations, especially fixations landing at the word beginning and leading to intraword forward saccades, increased with font size.

Obviously, visual acuity decreases with the eccentricity of parafoveal information. As long as a strong, above- 
threshold signal marks the end of the next word (such as a space between words in most alphabetic writing systems), low visual acuity about word endings may not be a serious problem for programming a saccade to the center of this word. Chinese readers, however, who must do without this salient word space signal, presumably compute word boundary on the basis of word or character frequency, taking into account how often they occur at the beginning or at the end of words (Richter, Yan, Engbert, \& Kliegl, 2010). Thus, for them precise, high-acuity information about the end of the parafoveal word is critical for word segmentation. Preventing readers from using such information decreases the possibility of selecting the word center as the saccade target and results in more first-of-multiple fixations at the word beginning.

Parafoveal word segmentation and saccade-target selection in Chinese reading

We interpret the results from the present study as further evidence against the 'fixed length saccade strategy' proposed by S.-N. Yang and McConkie (2004; see also Yang \& Vitu, 2007) who argued that word units do not serve as saccade targets. According to this claim and given that most Chinese words are of similar length, the PVL in Chinese reading emerges as a consequence of saccades being programmed with a constant amplitude (subject to random error). The decrease of relative saccade amplitude with increased font size is difficult to reconcile with this simple reading model.

Similar to results from reading of alphabetic scripts (McDonald \& Shillcock, 2004; Nuthmann \& Kliegl, 2009; Rayner, 1979), single-fixation PVL also located near the word center in Chinese reading, replicating the results of Yan et al. (2010). Moreover, in the present study, we were able to establish the word-centered PVL for four different font sizes. We submit this result as strong evidence for the proposition that readers of Chinese estimate/compute the position of the upcoming word in the parafovea and program a saccade to the center of the selected word.

For two-fixation cases, the landing position distributions exhibited peaks at the word beginning in each font-size condition, replicating again previous results from unspaced text reading (Kajii et al., 2001; Rayner et al., 1998; Sainio et al., 2007; Yan et al., 2010) and reflecting word segmentation difficulty when reading unspaced texts (Rayner et al., 1998). The result is consistent with our previous explanation that first-of-multiple fixations at the beginning of words are indicative of a default strategy of saccade-target selection when parafoveal word segmentation failed or was not completed in time (Yan et al., 2010).

In a recent study, Perea and Acha (2009) found reading cost in unspaced sentences relative to normally written sentences, with smaller cost for sentences with words presented in an alternating bold-normal unspaced series than for sentences with words presented as a regular unspaced series. These results indicate that readers can use other information than an empty space to determine word boundaries for target selection. In the case of Chinese script, readers may also use other information than spaces, such as the frequency of character co-occurrence, to compensate for the lack of space between words in order to complete word segmentation in the parafovea (Richter et al., 2010).

Results from the present study provide also some information about the role of visual complexity in parafoveal word segmentation. If the next word is of low visual complexity, parafoveal preprocessing increases the chance of successful word segmentation. In this case, readers are more likely to program a saccade that targets the word center, a position known to have the highest foveal processing efficiency (O'Regan \& Levy-Schoen, 1987). Thus, word processing is likely to be completed in a single fixation. On the other hand, if the next word is visually complex, segmentation is likely to fail (or delayed beyond target selection). In this case, the default saccade is the word beginning and a refixation is more likely to follow, too.

Systematic saccadic range effect and random oculomotor error

A decrease of text-based, relative saccade amplitude with increasing font size is compatible with the so-called range effect (Kapoula, 1985; Kapoula \& Robinson, 1986), that is the observation that the amount of undershoot of saccade targets increases with the distance of the saccade target. For example, Miellet et al. (2009) presented text with gazecontingent parafoveal magnification of font and argued that undershoots were more likely to occur in this condition because the saccade target was physically further away than during normal text reading. According to the range effect, more overshoots (intraword forward saccades) should be observed for the $0.4^{\circ}$ rather than the $0.7^{\circ}$ condition, and likewise, more undershoots (intraword backward saccades) should be observed for the $0.7^{\circ}$ rather than the $0.4^{\circ}$ condition. However, neither the percentages of intraword forward nor backward saccades differed between the $0.4^{\circ}$ and $0.7^{\circ}$ conditions. We explain these results as a consequence of equally valid information at the end of the to-be-fixated word for the two small font sizes. In these cases, visual acuity was unlikely to limit parafoveal word segmentation.

Refixations in alphabetic reading could be explained as a consequence of mislocated fixations (Nuthmann, Engbert, \& Kliegl, 2005) and two predictions can be derived from 
this random oculomotor error account; (1) a larger font size induces a greater spatial tolerance for random oculomotor error, implying fewer refixations with larger font, and (2) forward and backward refixations should occur roughly equally often. However, in the present study, (1) the overall percentage of refixations increased with font size, and (2) the percentage of forward refixations increased significantly between the $0.7^{\circ}$ font size and the largest font size by $7 \%$ whereas the percentage of backward refixations decreased significantly but only by $1.2 \%$. Neither of those results can be reconciled with the above predictions derived from the oculomotor error account and the mislocated fixation explanation is not compatible with the observed pattern of refixations in Chinese reading.

In summary, the higher percentage of fixations at the word beginning under large font size condition in Chinese reading is not due to a saccadic range effect or due to random oculomotor error. The results are compatible with what has been called the default strategy for readers encountering parafoveal segmentation difficulty (Yan et al., 2010).

\section{Conclusion}

We propose that due to the lack of spaces between words in Chinese script, increase of font size decreased relative saccade amplitude but did not harm the word-based saccade targeting in general. Results consolidated and extended a model about flexible saccade targeting as proposed by Yan et al. (2010). Of course, given the numerous differences between Chinese and alphabetic writing systems, the absence of interword spacing may not be the only characteristic of the Chinese script that contributes to the current finding. Much further work is still needed to establish a clear understanding of eye movement control of Chinese script.

Acknowledgements This research was supported by Deutsche Forschungsgemeinschaft (KL955/15) to Reinhold Kliegl and Ralf Engbert, by the China Postdoctoral Science Foundation (20080440008, 200902025) to Ming Yan, by Program for Changjiang Scholars and Innovative Research Team in University (IRT0710), by grants from the Natural Science Foundation of China (30870758) and from Beijing Educational Committee (SYS100270661). We thank Eike Richter and Jochen Laubrock for helpful comments. Hua Shu and Wei Zhou equally contributed to this work.

\section{References}

Bai, X. J., Yan, G. L., Liversedge, S. P., Zang, C. L., \& Rayner, K. (2008). Reading spaced and unspaced Chinese text: Evidence from eye movements. Journal of Experimental Psychology: Human Perception and Performance, 34(5), 1277-1287.
Bates, D. M., Maechler, M., \& Dai, B. (2008). Ime4: Linear mixedeffects models using S4 classes. R package version 0.999375-24.

Beijing Language Institute Publisher (1986). Modern Chinese Word Frequency Dictionary (in Chinese). Beijing: Beijing Language Institute Publisher.

Bullimore, M. A., \& Bailey, I. L. (1995). Reading and eye movements in age-related maculopathy. Optometry and Vision Science, 72, $125-138$

Engbert, R., \& Kliegl, R. (2003). Microsaccades uncover the orientation of covert attention. Vision Research, 43(9), 10351045 .

Engbert, R., \& Krügel, A. (2010). Readers use Bayesian estimation for eye movement control. Psychological Science, 21, 366-371.

Epelboim, J., Booth, J. R., Ashkenazy, R., Taleghani, A., \& Steinman, R. M. (1997). Fillers and spaces in text: The importance of word recognition during reading. Vision Research, 37(20), 2899-2914.

Epelboim, J., Booth, J. R., \& Steinman, R. M. (1994). Reading unspaced text: Implications for theories of reading eye movements. Vision Research, 34(13), 1735-1766.

Hsu, S.-H., \& Huang, K.-C. (2000a). Effects of word spacing on reading Chinese text from a video display terminal. Perceptual and Motor Skills, 90, 81-92.

Hsu, S.-H., \& Huang, K.-C. (2000b). Interword spacing in Chinese text layout. Perceptual and Motor Skills, 91, 355-365.

Kajii, N., Nazir, T. A., \& Osaka, N. (2001). Eye movement control in reading unspaced text: The case of the Japanese script. Vision Research, 41(19), 2503-2510.

Kapoula, Z. A. (1985). Evidence for a range effect in the visual system. Vision Research, 25, 1155-1157.

Kapoula, Z. A., \& Robinson, D. A. (1986). Saccadic undershoot is not inevitable: Saccades can be accurate. Vision Research, 26, 735743.

Kliegl, R., Grabner, E., Rolfs, M., \& Engbert, R. (2004). Length, frequency, and predictability effects of words on eye movements in reading. European Journal of Cognitive Psychology, 16, 262 284.

Kliegl, R., Yan, M., Pan, J., Richter, E., Engbert, R., \& Shu, H. (2009, September). Current issues in research on eye movements in reading. Paper presented at the Symposium on Eye Movements, EEG, and Their Co-registration During Reading and Related Processes, Beijing, China.

Liu, L., Klein, S. A., Xue, F., Zhang, J., \& Yu, C. (2009). Using geometric moments to explain human letterrecognition near the acuity limit. Journal of Vision, 9(1), 1-18. doi:10.1167/ 9.1.26

McConkie, G. W., Kerr, P. W., Reddix, M. D., \& Zola, D. (1988). Eye movement control during reading: I. The location of initial eye fixations in words. Vision Research, 28, 1107-1118.

McDonald, S. A., \& Shillcock, R. C. (2004). The potential contribution of preplanned refixations to the preferred viewing location. Perception \& Psychophysics, 66(6), 1033-1044.

Miellet, S., O'Donnell, P. J., \& Sereno, S. C. (2009). Parafoveal magnication visual acuity does not modulate the perceptual span in reading. Psychological Science, 20(6), 721-728.

Mohammed, Z., \& Dickinson, C. M. (2000). The interrelationship between magnification, field of view and contrast reserve: The effect on reading performance. Ophthalmic \& Physiological Optics, 20, 464-472.

Morrison, R. E., \& Rayner, K. (1981). Saccade size in reading depends upon character spaces and not visual angle. Perception \& Psychophysics, 30(4), 395-396.

Nuthmann, A., Engbert, R., \& Kliegl, R. (2005). Mislocated fixations during reading and the inverted optimal viewing position effect. Vision Research, 45(17), 2201-2217.

Nuthmann, A., \& Kliegl, R. (2009). Preferred viewing locations: a validation and an extension. Perception, 38(6), 901-902. 
O'Regan, J. K. (1979). Eye guidance in reading: Evidence for the linguistic control hypothesis. Perception \& Psychophysics, 25, 501-509.

O'Regan, J. K. (1980). The control of saccade size and fixation duration during reading: The limits of linguistic control. Perception \& Psychophysics, 28, 112-117.

O'Regan, J. K. (1983). Elementary perceptual and eye movement control processes in reading. In K. Rayner (Ed.), Eye movements in reading: Perceptual and language processes (pp. 121-139). New York: Academic Press.

O'Regan, J. K. (1990). Eye movements in reading. In E. Kowler (Ed.), Eye movements and their role in visual and cognitive processes (pp. 395-453). Amsterdam: Elsevier.

O'Regan, J. K., \& Levy-Schoen, A. (1987). Eye movement strategy and tactics in word recognition and reading. In M. Coltheart (Ed.), Attention and performance: Vol. 12. The psychology of reading (pp. 363-383). Hillsdale: Erlbaum.

Perea, M., \& Acha, J. (2009). Space information is important for reading. Vision Research, 49, 1994-2000.

R Development Core Team (2008). R: A language and environment for statistical computing. R Foundation for Statistical Computing, Vienna, Austria. ISBN 3-900051-07-0. Retrieved from http:// www.R-project.org

Rayner, K. (1979). Eye guidance in reading: fixation locations within words. Perception \& Psychophysics, 8, 21-30.

Rayner, K., Fischer, M. H., \& Pollatsek, A. (1998). Unspaced text interferes with both word identification and eye movement control. Vision Research, 38(8), 1129-1144.

Rayner, K., \& Pollatsek, A. (1996). Reading unspaced text is not easy: Comments on the implications of Epelboim et al.'s (1994) study for models of eye movement control in reading. Vision Research, 36(3), 461-465.

Reichle, E. D., Rayner, K., \& Pollatsek, A. (1999). Eye movement control in reading: Accounting for initial fixation locations and refixations within the E-Z Reader model. Vision Research, 39, 4403-4411.

Richter E., Yan, M., Engbert R., \& Kliegl, R. (2010). Modeling Chinese reading with SWIFT: How does word segmentation affect targeting? Paper presented at the 4th China International Conference on Eye Movements, Tianjin, China.

Sainio, M., Hyönä, J., Bingushi, K., \& Bertram, R. (2007). Vision Research, 47, 2575-2584.

$\mathrm{Xu}$, M. Y., \& Jordan, T. (2009). Assessing effects of viewing distance on normal Chinese reading: Some methodological and theoretical implications. Behavior Research Methods, 41(4), 971-976.

Yan, M., Kliegl, R., Richter, E. M., Nuthmann, A., \& Shu, H. (2010). Flexible saccade-target selection in Chinese reading. The Quarterly Journal of Experimental Psychology, 63, 705-725. doi:10.1080/17470210903114858

Yang, S.-N., \& McConkie, G. W. (2004). Saccade generation during reading: are words necessary? European Journal of Cognitive Psychology, 16, 226-261.

Yang, S.-N., \& Vitu, F. (2007). Dynamic coding of saccade length in reading. In R. P. G. van Gompel, M. H. Fischer, W. S. Murray, \& R. L. Hill (Eds.), Eye movements: A window on mind and brain (pp. 293-317). Amsterdam: Elsevier.

Yu, B., Zhang, W., Jing, Q., Peng, R., Zhang, G., \& Simon, H. A. (1985). STM capacity for Chinese and English language materials. Memory \& Cognition, 13, 202-207. 